\title{
Efficient Multi-Stage Time Marching for Viscous Flows via Local Preconditioning
}

\author{
William L. Kleb;, William A. Wood* \\ NASA Langley Research Center, Hampton, VA 23681. \\ and \\ Bram van Leer ${ }^{\dagger}$ \\ The University of Michigan, Ann Arbor, MI 48109
}

\begin{abstract}
A new method has been developed to accelerate the convergence of explicit timemarching, laminar, Navier-Stokes codes through the combination of local preconditioning and multi-stage time marching optimization. Local preconditioning is a technique to modify the time-dependent equations so that all information moves or decays at nearly the same rate, thus relieving the stiffness for a system of equations. Multi-stage time marching can be optimized by modifying its coefficients to account for the presence of viscous terms, allowing larger time steps. We show it is possible to optimize the time marching scheme for a wide range of cell Reynolds numbers for the scalar advectiondiffusion equation, and local preconditioning allows this optimization to be applied to the Navier-Stokes equations. Convergence acceleration of the new method is demonstrated through numerical experiments with circular advection and laminar boundary-layer flow over a flat plate.
\end{abstract}

\section{Nomenclature}

$\begin{array}{ll}\text { Roman letters } \\ A & \text { Cell area } \\ a, b & \text { Horizontal and vertical components of } \\ & \text { advection velocity, } q \\ c & \text { Speed of sound } \\ \mathrm{C} & \text { Viscous flux Jacobian in the } x \text {-direction } \\ C_{n} & \text { Condition number, ratio of largest to } \\ & \text { smallest eigenvalues } \\ d & \text { Horizontal shift for Tchebyshev polynomial } \\ & \text { transformation } \\ E & \text { Energy per unit volume } \\ \mathrm{E} & \text { Viscous flux Jacobian in the } y \text {-direction } \\ \mathbf{F} & \text { Inviscid flux vector, } x \text {-component } \\ \mathbf{G} & \text { Inviscid flux vector, } y \text {-component } \\ H & \text { Total enthalpy per unit volume } \\ h & \text { Ratio of cell area to the length of the } \\ \mathbf{H} & \text { diagonal } \\ \mathbf{J} & \text { Inviscid flux vector }\end{array}$

*Aerospace Engineer, Aerothermodynamics Branch, Aeroand Gas-Dynamics Division.

†Professor, Department of Aerospace Engineering, AIAA fellow.

Copyright (c) 1999 by the American Institute of Aeronautics and Astronautics, Inc. No copyright is asserted in the United States under Title 17, U.S. Code. The U.S. Government has a royaltyfree license to exercise all rights under the copyright claimed herein for Governmental Purposes. All other rights are reserved by the copyright owner. $l \quad$ Length from a cell corner to the diagonal along the advection velocity direction

$L_{0} \quad$ Reference length

$M \quad$ Mach number

$p \quad$ Pressure

$\mathrm{P} \quad$ Preconditioning matrix

$P_{n} \quad N^{t h}$-order polynomial

Pr Prandtl number, 0.72

$q \quad$ Advection velocity

$Q_{0} \quad$ Reference velocity

$q_{x, y} \quad$ Heat flux components

R Viscous flux vector, $x$-component

$R_{S} \quad$ Negative Real extent of the spatial operator's Fourier Footprint

$R_{T} \quad$ Negative Real extent of the temporal operator

Re Reynolds number

$R e_{\Delta} \quad$ Cell Rènolds number (generic)

$R e_{\Delta x}$ Cell Reynolds number based on $\Delta x$

$R e_{h} \quad$ Cell Reynolds number based on $h$

$R e_{x} \quad$ Reynolds number based on $x$-coordinate

Res Residual

$S \quad$ Conserved scalar quantity

$s \quad$ Cell length

S Viscous flux vector, $y$-component

$T$ Temperature

$T_{n} \quad N^{\text {th }}$-order Tchebyshev polynomial

$U \quad$ Blasius freestream velocity 


$\begin{array}{ll}u & \text { Velocity, horizontal component } \\ \mathrm{U} & \text { Vector of conserved variables } \\ v & \text { Velocity, vertical component } \\ x & \text { Horizontal coordinate direction } \\ y & \text { Vertical coordinate direction } \\ z & \text { Complex number }\end{array}$

\section{Subscripts}

( ) 0 Reference quantity

( ) $E \quad$ Euler

( ) $)_{i} \quad$ Cell face index

()$_{j} \quad$ Cell index in $y$-direction

()$_{N S}$ Navier-Stokes

( ) $t \quad$ Derivative with respect to time

()$_{x, y}$ Horizontal and vertical components or derivatives

\section{Conventions \\ () Non-dimensional quantity (briefly) \\ $d$ ( ) Differential quantity}

\section{Symbols}

$\alpha_{k} \quad$ Runge-Kutta coefficient for stage $k$

$\beta \quad$ Fourier frequency

$\Delta t \quad$ Time step

$\Delta x \quad$ Horizontal grid spacing

$\Delta y \quad$ Vertical grid spacing

$\varepsilon \quad$ Scaling for Tchebyshev polynomial transformation

$\eta \quad$ Blasius boundary layer coordinate

$\gamma \quad$ Ratio of specific heats, 1.4

$\Im$ Imaginary component

$\kappa \quad$ Parameter which controls upwind-biasing

$\mu \quad$ Kinematic viscosity

$\mu_{0} \quad$ Reference kinematic viscosity

$\nu \quad$ Courant number

$\phi \quad$ Flow angle with respect to the horizontal

$\Re$ Real component

$\rho \quad$ Mass density

$\varrho \quad$ Prandtl-Glauert factor

$\sigma \quad$ Von Neumann number

$\varsigma \quad$ Cell stretching parameter

$\sigma_{\pi} \quad$ High frequency damping

$\tau_{i j} \quad$ Shear stress components

$\theta \quad$ Angle between $h$ and the advection velocity

$\xi \quad$ High-frequency scaling for Preconditioner

$\zeta \quad$ Transformed complex number

$\boldsymbol{A} \quad$ Cell aspect ratio

$\boldsymbol{R}_{q} \quad$ Equivalent cell aspect ratio in the flow direction

D Common denominator

\section{$\mathcal{F} \quad$ Fourier Footprint}

\section{Introduction}

7 HIS work is motivated by the poor convergence of explicit time-stepping Computational Fluid Dynamics (CFD) codes for viscous flow problems. The recent development of local preconditioning methods $^{1-4}$ and the flexibility of multistage time-marching schemes provide the tools to relieve this bottleneck. ${ }^{\mathrm{a}}$ With the strong shift toward cache-based parallel architectures, implicit schemes are beginning to show limitations for this emerging environment.

Typically an explicit time marching schome is limited by the minimum of an inviscid time $\operatorname{step}^{5,6}$ and a viscous time step. ${ }^{7}$ The inviscid portion relates to the advective part of the equations and the viscous portion represents the dissipation component. In terms of Von Neumann stability analysis ${ }^{8}$ these portions correspond to the extents of the Fourier Footprint ${ }^{b}$ along the imaginary axis and negative real axis, respectively.

In the beginning of the "Euler era" (early 1980s), multistage time-marching schemes were optimized for the maximum imaginary extent of the stability domain, given the number of stages of the scheme. ${ }^{9}$ This allows inviscid problems to be solved efficiently on a single grid. Very soon, multigrid relaxation made its entrance, ${ }^{10}$ and the emphasis in multi-stage design shifted to choosing the coefficients such that maximum high-frequency damping results for a given number of stages, typically at half the maximum allowable time step. ${ }^{11-13}$ This optimization technique originally was based on a scalar analysis, losing most of its validity when applied to a system of equations. The introduction of local preconditioning for the Euler equations, ${ }^{4}$ which tends to equalize the advective time scales and concentrates the Fourier footprint, finally made it possible to optimize high-frequency damping for all types of waves admitted by the inviscid system. ${ }^{14-16}$

For a typical viscous flow problem, however, a majority of the computational cells are limited in the size of their time step by the viscous criterion, due to the limitcd extent of the stability boundary along the negative real axis. By making the multistage coefficients a function of the local cell Reynolds number, $R e_{\Delta}$, the stability boundary can be reshaped to alleviate this restriction. ${ }^{15,16}$

For systems of equations, local preconditioning is necessary for equalizing various time scales admitted by the system..$^{16-20}$ However, the analytical preconditioning analysis for the Navier-Stokes equations is much more complicated than for the Euler equations, and is still far from complete. As a result, the current paper uses a simple block-Jacobi method to extend the

\footnotetext{
a Also, as a fringe benefit of local preconditioning, the solution accuracy at low Mach numbers is enhanced.

b The locus of the Fourier transform in the complex plane.
} 
Euler preconditioner to viscous problems. ${ }^{21}$

In the work of Lynn, ${ }^{15,16}$ multistage methods are optimized based on the precise Fourier footprint of the spatial operator. The optimization must be performed separately for each choice of cell Reynolds number. The multistage time steps can be tabulated as functions of $R e_{\Delta}$, or, more practically, given as functions that closely fit the data. Neither Lynn nor D. Lee ${ }^{18}$ actually carry out Navier-Stokes calculations with $R e_{\Delta^{-}}$ dependent multistage coefficients.

In the present work the optimization procedure is reversed, and thereby greatly simplified. We start with a family of multistage schemes that has stability domains of desirable shape. Then, using the scalar advection-diffusion operator as a foothold, we fit the spatial footprint within the temporal stability boundary to achieve stability and possibly some other desirable property, like prescribed high-frequency damping. Related work which optimizes the time step without considcration for the associated damping is given by Lorber et $a .^{22,23}$ The transition from the advectiondiffusion operator to the Navier-Stokes operator is accommodated by the local preconditioning. This procedure provides a numerical relationship between $R e_{\Delta}$ and the multistage time step coefficients. The resulting $R e_{\Delta}$-dependent marching schemes are used to solve the advection-diffusion equation and compute laminar boundary-layer flow.

\section{Runge-Kutta Design}

Optimizing the time-marching scheme for efficiency or high-frequency damping is most easily accomplished by working in the complex space of the Fourier transform via Von Neumann stability analysis. ${ }^{8}$ The overriding goal, of course, is stability, i.e., to contain the Fourier Footprint of the spatial discretization inside the stability boundary of the time-marching scheme. A secondary goal is to provide some level of highfrequency damping. If only single-grid marching is desired, the tradition is to go for the largest time step possible. For multigrid marching it is necessary to maximize high-frequency damping, which means giving-up the maximum time step. The latter strategy actually turns out to also be more efficient for singlegrid marching, as was shown by Tai. ${ }^{13}$ Maximizing high-frequency damping without setting a target level for the damping, though, is not a good idea for a viscous equation solver, as this tends to reduce the time step to unacceptably low values. ${ }^{15,16}$

For the sake of simplicity we will start our analysis by examining the scalar model equation for the NavierStokes system of equations: the advection-diffusion equation, ${ }^{c}$

$$
S_{t}+a S_{x}+\iota S_{y}=\frac{1}{R e}\left(S_{x x}+S_{y y}\right)
$$

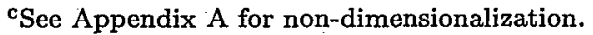

where $S$ is some conserved, scalar quantity; $a$ and $b$ are horizontal and vertical components of the advection velocity, respectively; and $R e$ is the Reynolds number.

Once we choose a discretization of the spatial terms, we are stuck with the resulting Fourier Footprint, so we will study it first. Afterward we will examine the temporal operator, and finally, the procedure used to fit one to the other.

\section{Spatial Operator}

For this study we will use central differencing for the viscous terms and the $\kappa$-scheme $e^{24,25}$ for the advective terms. This yields,

$$
\begin{aligned}
\mathcal{F}= & -\left((1-\kappa) \frac{a \Delta t}{\Delta x}+2 \frac{\Delta t}{R e \Delta x^{2}}\right)\left(1-\cos \beta_{x}\right) \\
& -\frac{(1-\kappa)}{a} \frac{a \Delta t}{\Delta x}\left(1-\cos 2 \beta_{x}\right) \\
& -i \frac{a \Delta t}{\Delta x} \sin \beta_{x}+i \frac{(\kappa-1)}{4} \frac{a \Delta t}{\Delta x} \sin 2 \beta_{x} \\
& -\left((1-\kappa) \frac{b \Delta t}{\Delta y}+2 \frac{\Delta t}{R e \Delta y^{2}}\right)\left(1-\cos \beta_{y}\right) \\
& -\frac{(1-\kappa)}{4} \frac{b \Delta t}{\Delta y}\left(1-\cos 2 \beta_{y}\right) \\
& -i \frac{b \Delta t}{\Delta y} \sin \beta_{y}+i \frac{(\kappa-1)}{4} \frac{b \Delta t}{\Delta y} \sin 2 \beta_{y}
\end{aligned}
$$

where $\kappa \in[-1,1]$, providing a blending between fully upwind and central differencing, respectively. In a moment we will look at the resulting Fourier Footprint, but first let us define some parameters,

$$
\begin{aligned}
q & =\sqrt{a^{2}+b^{2}}, & & \text { the advection speed, } \\
\phi & =\arctan \frac{b}{a}, & & \text { the flow angle, } \\
\boldsymbol{R} & =\frac{\Delta x}{\Delta y}, & & \text { cell aspect ratio, }
\end{aligned}
$$

and scale the footprint by fixing the position of the high frequency components $\left(\beta_{x, y}=\pi\right)$ at $-R_{S}$. With these definitions, Eq. 2 becomes,

$$
\begin{array}{r}
-R_{S}=-2 \Delta t\left[(1-\kappa) \frac{q}{\Delta x}(\cos \phi+A \sin \phi)\right. \\
\left.+\frac{2}{R e \Delta x^{2}}\left(1+R^{2}\right)\right] .
\end{array}
$$

In this form, two length scales have emerged,

$$
h=\frac{\Delta x}{\sqrt{1+\overline{A R}^{2}}},
$$

the ratio of cell area to the length of the diagonal, and

$$
l=\frac{\Delta x}{\cos \phi+A \sin \phi},
$$

which is the length from a cell corner to the diagonal along the advection velocity direction. Figure 1 


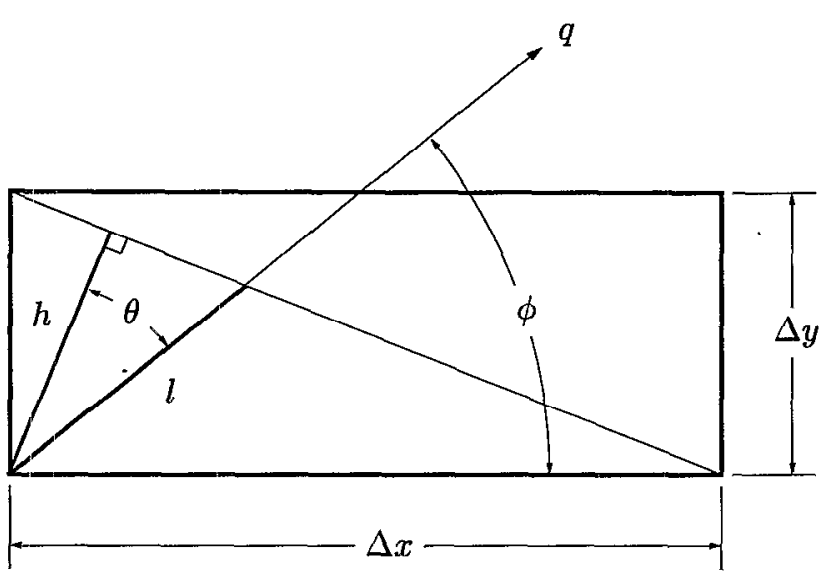

Fig. 1 Time-step cell and flow geometry.

shows the cell geometry and the associated lengths. Moreover, $h$ and $l$ are related by $l=h / \cos \theta$ where $\theta$ is the angle between $h$ and the advection velocity.

Using the new length scale, $h$, we solve for $\Delta t$ to fix the high frequency portion of the footprint at $-R_{s}$,

$$
\Delta t=\frac{\frac{R_{S}}{2}}{\frac{q}{h}(1-\kappa) \cos \theta+\frac{2}{R e h^{2}}} .
$$

Factoring out the $q / h$ and converting the Reynolds number, $R e$, to the cell Reynolds number, $R e_{h}$, we find,

$$
\Delta t=\frac{R_{S}}{2} \frac{h}{q} \frac{R e_{h}}{R e_{h}(1-\kappa) \cos \theta+2} .
$$

Substituting this time step into Eq. 2 gives our scaled Fourier Footprint of the form,

$$
\begin{aligned}
\mathcal{F}=-\frac{R{ }_{S}}{2} \frac{h}{\Delta x} \frac{R e_{h}}{R e_{h} \cos \theta+2} & \\
& {\left[\left(\frac{R e_{\Delta x} \cos \phi+2}{R e_{h}}\right)\left(1-\cos \beta_{x}\right)+i \cos \phi \sin \beta_{x}\right.} \\
& +A R\left(\frac{R e_{\Delta x} \sin \phi+2}{R e_{h}}\right)\left(1-\cos \beta_{y}\right) \\
& \left.+i R \sin \phi \sin \beta_{y}\right] .
\end{aligned}
$$

Finally, we reveal the Fourier Footprint in Fig. 2 which shows the effect of various $\kappa$ 's for $R_{S}=1, A R=1$, and $R e=1$. Defining a non-dimensional time step for the advection-diffusion equation is not trivial, see Appendix B for more details.

Now that we have the spatial operator's footprint, we turn our attention to the temporal stability boundary.

\section{'Temporal Scheme}

For this study the stability boundary is given by a transformation of Tchebyshev polynomials attributed to Manteuffel, ${ }^{26}$

$$
P_{n}(z)=\frac{T_{n}\left(\frac{d-z}{\varepsilon}\right)}{T_{n}\left(\frac{d}{\varepsilon}\right)}
$$
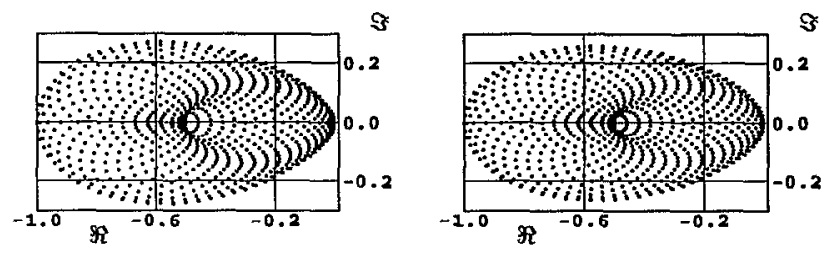

a) Upwind $(\kappa=-1)$.

b) Fromm's scheme $(\kappa=0)$.
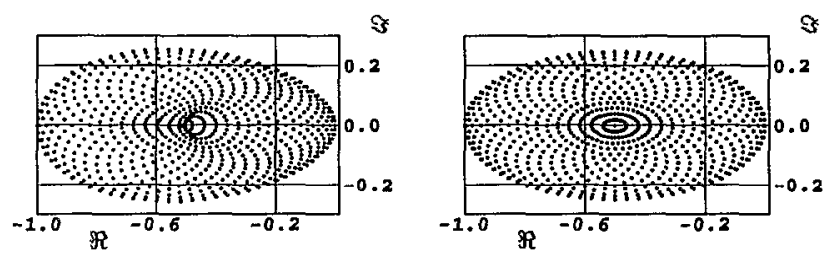

c) Third-order in $1 D$ only $(\kappa=1 / 3)$.

d) Central difference $(\kappa=1)$.

Fig. 2 Effect of $\kappa$ on the footprint of the higher-order scheme $\left(A R=1, \phi=45 \mathrm{deg}, R e_{h}=1, \beta_{x}, \beta_{y} \in[0,2 \pi]\right.$, $\Delta \beta=2 \pi / 40)$.

where $n$ is the order of the respective polynomials, $z$ is a number in the left complex plane, and the sequence of Tchebyshev polynomials are given by recursion,

$$
\begin{aligned}
T_{0}(\zeta) & =1 \\
T_{1}(\zeta) & =\zeta \\
T_{n+1}(\zeta) & =2 \zeta T_{n}(\zeta)-T_{n-1}(\zeta), n>1
\end{aligned}
$$

The resulting coefficients of $P_{n}(z)$ are chosen such that the $\left\{P_{n}(z) \mid=1\right.$ stability boundary remains simply connected and the blended polynomial is scaled properly, i.e.,

$$
P_{n}(0)=1
$$

and

$$
\left.\frac{d P_{n}}{d z}\right|_{z=0}=1
$$

For example, a four stage scheme would take the form,

$$
\begin{aligned}
P_{4}(z)= & 1-\frac{32 d^{3}-16 \varepsilon^{2} d}{\mathcal{D}} z \\
& +\frac{48 d^{2}-8 \varepsilon^{2}}{\mathcal{D}} z^{2}-\frac{32 d}{\mathcal{D}} z^{3}+\frac{8}{\mathcal{D}} z^{4}
\end{aligned}
$$

where $\mathcal{D}=8 d^{4}-8 d^{2} \varepsilon^{2}+\varepsilon^{4}$. To satisfy the conditions given by Eqs. 11 and 12 we find,

$$
\varepsilon=\sqrt{4 d(2+d)+\sqrt{16 d^{2}(2+d)^{2}-8 d^{3}(d+4)}}
$$

Thus, we now have a one-parameter family of stability boundaries governed by $d$.

Next, we recall that a 4-stage Runge-Kutta scheme has the form of

$P_{4}(z)=1+\alpha_{4} z+\alpha_{4} \alpha_{3} z^{2}+\alpha_{4} \alpha_{3} \alpha_{2} z^{3}+\alpha_{4} \alpha_{3} \alpha_{2} \alpha_{1} z^{4}$ 
So by equating the coefficients in Eqs. 13 and 15 we can determine the coefficients, $\alpha_{k}$, for the corresponding Runge-Kutta scheme.

\section{Fitting}

The procedure employed is analogous to blowing up a balloon into a bear trap: a given stability boundary (the bear trap) is chosen; next, a given high frequency damping level is 'located on the negative real axis; and then the cell Reynolds number is increased until some part of the spatial operator's Fourier Footprint (the balloon) reaches a specified damping contour of the temporal operator. Figure 3 depicts the parameters, the stability boundary, and the spatial operator Fourier Footprint:

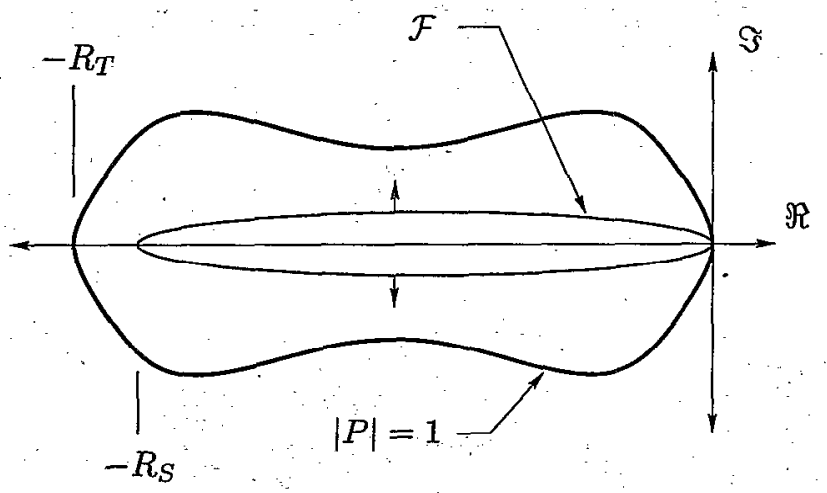

Fig. 3 Sketch of optimization procedure.

The fitting algorithm is as follows:

1. Pick a value for $d$. This sets the shape and extent of the Tchebyshev stability boundary.

2. Compute $-R_{T}$, the negative Real extent of the Temporal operator. (Hint: $R_{T}=2 d$.)

3. Given the negative Real extent of the stability domain, $-R_{T}$, as a starting point, find the negative Real extent of the spatial operator, $-R_{s}$, which satisfies the prescribed high frequency damping, ${ }^{d}$ $\sigma_{\pi}$, by moving toward the origin along the Real axis, computing $\left|P_{n}(z)\right|$.

4. Using the viscous limit $\left(R e_{h} \rightarrow 0\right)$ as an initial guess increase the cell Reynolds number until the prescribed damping conditions for the entire footprint or its high-frequency part are met.

Figure 4 shows a sequence of stability plots for different values of $d$. Superimposed are the spatial operator Fourier Footprinits which yield the maximum time step given a high-high frequency damping of 0.5 . The contours in Fig. 4 represent levels of the temporal operator's amplification (or damping) factor, $|P|$, from 0.1 to 1.0 in increments of 0.1 .

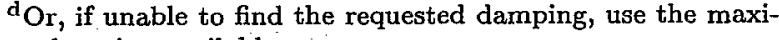
mum damping available.
}

Notice the large negative extent of the stability domain along the real axis, made possible by the Tchebyshev polynomial. The domain maximally extends to $-2 n^{2}$ for an $n$-stage scheme; however, for this choice the scheme is only stable in the limit of $\operatorname{Re}_{\Delta}=0$ (refer to Fig. 4(d)).

Figure 5 shows the reference time step and RungeKutta coefficients as a function of the cell Reynolds number for the resulting 4-stage Runge-Kutta scheme. This provides the link between the cell Reynolds number, the time step, and the multistage coefficients for a given cell. Note that the time step maintains an appreciable value all the way down to $R e_{h}=0.1$, which is smaller than many people use to resolve the boundary layer in a Navier-Stokes calculation.

\section{Extension to Systems (Local Preconditioning)}

Local preconditioning is a technique to remove stiffness from a system of equations. In the aeronautical CFD community, the set of timedependent, Reynolds-averaged, compressible, NavierStokes equations-sometimes simplified to the (inviscid) Euler equations - are typically solved in an iterative fashion to achieve a steady-state solution. Although in most cases only the steady-state solution is desired, the time-dependent equations are still employed so that the marching problem is well posed for all Mach numbers. The time-dependent Euler equations are hyperbolic and admit real wave-like fundamental solutions; the Navier-Stokes equations are mixed parabolic-hyperbolic and admit damped traveling waves as well as non-propagating damped modes.

Convergence to steady-state for inviscid calculations is impaired in some flow regimes due to the spread in the characteristic wave speeds. The spread is largest for waves moving in the streamwise direction, in which case their speeds are $q, q+c$, and $q-c$, where $q$ is the total flow speed and $c$ is the speed of sound. The ratio of the largest to smallest wave speed is termed the condition number, $C_{n}$, and serves as a measure of "stiffness". Figure 6 shows the condition number as a function of Mach number for the Euler equations. The solid line represents the original Euler equations and it is apparent that infinite stiffness occurs in both the subsonic and transonic regimes; as the Mach number further increases, the condition number asymptotes to the ideal value of one.

The dashed line in Fig. 6 rcpresents the Euler equations preconditioned with the Van Leer-Lee-Roe preconditioner. ${ }^{4}$ This preconditioner comes closest to achieving equalization of wave speeds, without affecting their direction of propagation or their hyperbolicity. The dashed line shows it is possible to eliminate completely the stiffness as the Mach number goes to zero, greatly reduce the transonic stiffness, and, in general, substantially lower the condition number for the system of equations. This makes the system behave as 


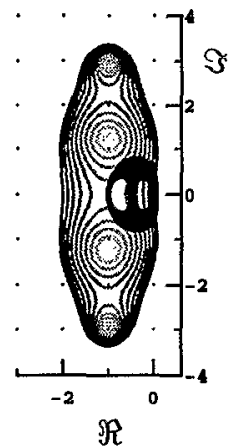

a) $d=-1$

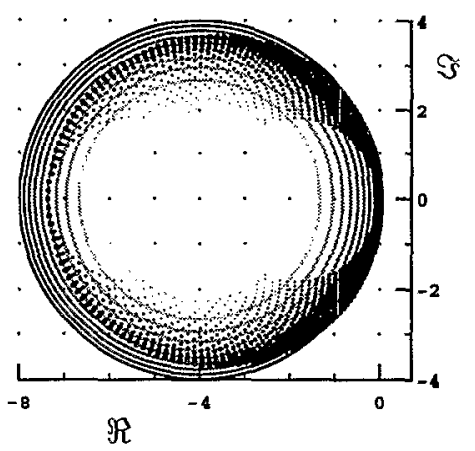

b) $d=-4$

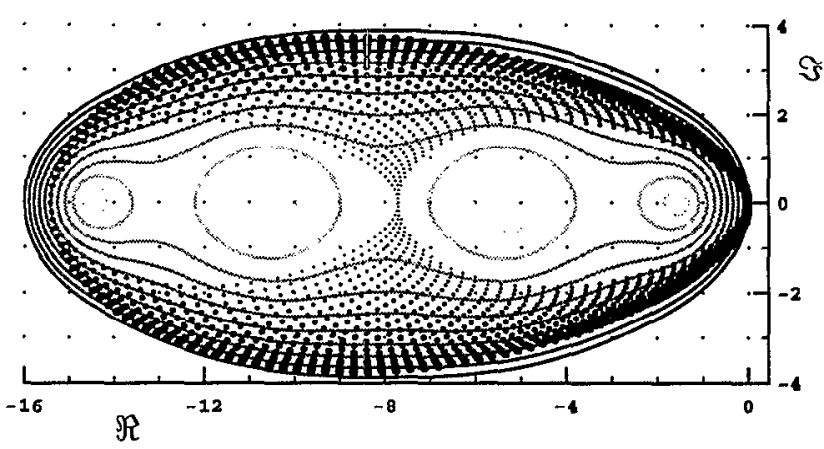

c) $d=-8$

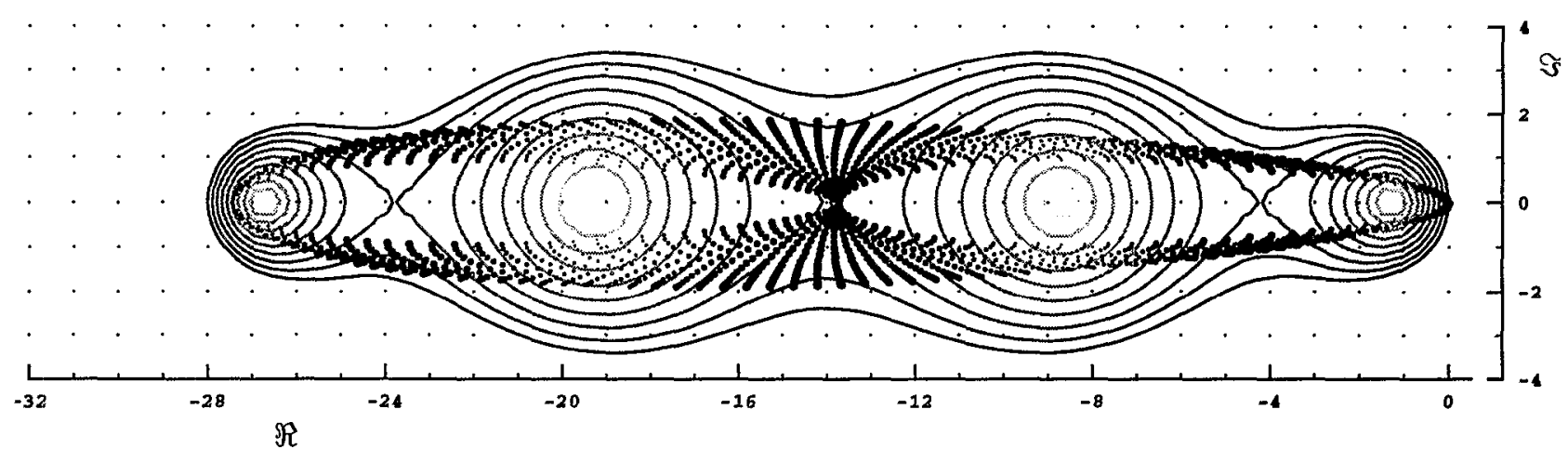

d) $d=-14$

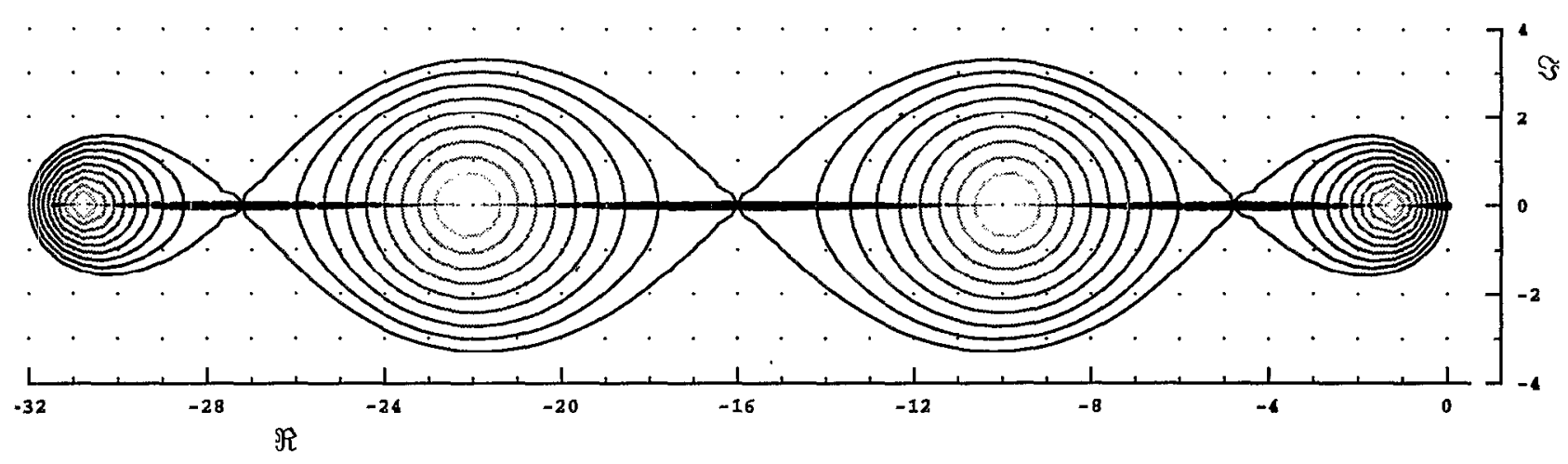

e) $d=-16$

Fig. 4 Optimized stability plots as a function of the temporal operator parameter, $d$, for Fromm's Scheme $(\kappa=0)$ with a prescribed high frequency damping of 0.5 with contours of damping every 0.1 and the size of the frequency symbols scaled by their respective damping. 


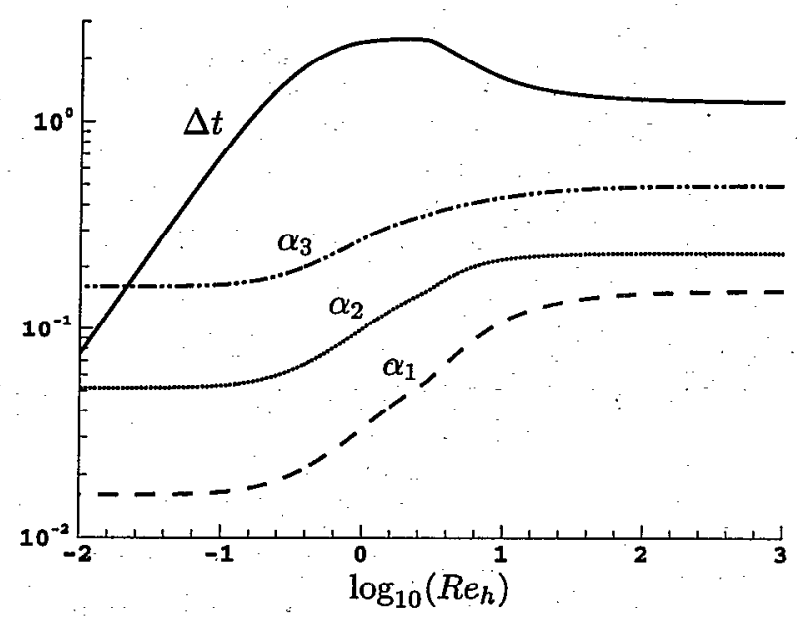

Fig. 5 The reference time step and Runge-Kutta coefficients as a function of the cell Reynolds number for the fitting presented in Fig. 4.

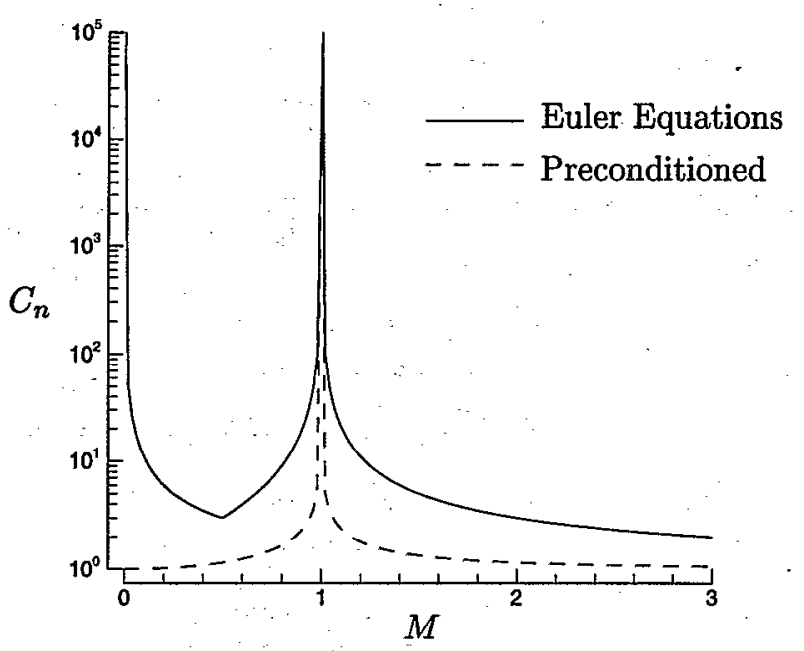

Fig. 6 Condition number as a function of Mach number for the Euler equations.

a scalar equation which has only a single wave speed, so we can apply the Runge-Kutta scheme developed in the previous section after accounting for some scaling factors.

The inviscid portion of local preconditioning used in this paper is that of Van Leer-Lee-Roe ${ }^{4}$ with a modification according to D. Lee ${ }^{20,27}$ and Lynn. ${ }^{16}$ In stream-aligned, hyperbolic symmetrizing variables, $\left[d p /(\rho c), d u, d v, d p-c^{2} d \rho\right]^{T}$, it has the form,

$$
P_{E}=\left[\begin{array}{cccc}
\xi \frac{M^{2}}{\varrho^{2}} & -\xi \frac{M}{\varrho^{2}} & 0 & 0 \\
-\frac{M}{\varrho^{2}} & 1+\frac{\xi}{\varrho^{2}} & 0 & 0 \\
0 & 0 & \xi & 0 \\
0 & 0 & 0 & 1
\end{array}\right]
$$

with $\varrho=\sqrt{\left|1-M^{2}\right|}$ and $\xi=\varrho /\left(\varrho+\mathbb{R}_{q}\right)$, where $\boldsymbol{R}_{q}$ is an effective streamwise aspect ratio given by summing the appropriate velocity projections for each cell face, $i$,

$$
\boldsymbol{R}_{q}=\frac{\sum_{i}\left|u \Delta x_{i}+v \Delta y_{i}\right|}{\sum_{i}\left|v \Delta x_{i}-u \Delta y_{i}\right|}
$$

This form is duc to Darmofal. ${ }^{28}$

Preconditioning of the Navier-Stokes equations is accomplished according to the method proposed in Ref. 21, namely, the addition of the viscous Jacobians:

$$
\mathrm{P}_{N S}^{-1}=\mathrm{P}_{E}^{-1}+\frac{2}{\Delta x(1-\kappa)}(\mathrm{C}+\mathrm{AE}),
$$

where $\mathrm{C}$ and $\mathrm{E}$ are the Jacobians of the viscous fluxes in the $x$ and $y$ directions, respectively.

. Thus, instead of marching to the steady state with

$$
\mathrm{U}_{t}=\operatorname{Res}(\mathrm{U}),
$$

march with a preconditioned residual

$$
\mathbf{U}_{t}=\mathrm{P}(\mathbf{U}) \operatorname{Res}(\mathbf{U}) .
$$

This can be viewed as marching with a matrix time step as opposed to a scalar time step. However, the following three elements of an existing code need to be modified to account for the new, preconditioned system:

- Time-step definition

- Artificial dissipation

- Boundary conditions

First, since preconditioning serves to collapse all wave speeds to the total flow speed, $q$, the time step should be based on the flow speed, $q$, and not the largest acoustic wave speed, $q+c$, which is typically used in the unconditioned case.

Next, for an upwind scheme the artificial dissipation matrices $|\mathrm{A}|$ and $|\mathrm{B}|$ need to be evaluated in terms of preconditioned quantities, i.e., they become $\mathrm{P}^{-1}|\mathrm{PA}|$ and $\mathrm{P}^{-1}|\mathrm{~PB}|$ for the preconditioned system.

And lastly, if one is employing "weak" (image cell) boundary conditions and using the modified upwind scheme to solve for the boundary fluxes, no change is necessary for the boundary conditions. However, if one is using explicit characteristic boundary conditions and/or employs "strong", specified boundary fluxes, these procedures need to be modified to account for the preconditioned equations that are now being solved.

\section{Numerical Experiments}

Two numerical experiments were performed, the first on the scalar model equation for the Navier-Stokes equations: advection-diffusion; and the second experiment solved the Navier-Stokes system of equations for boundary layer flow over a flat plate. 


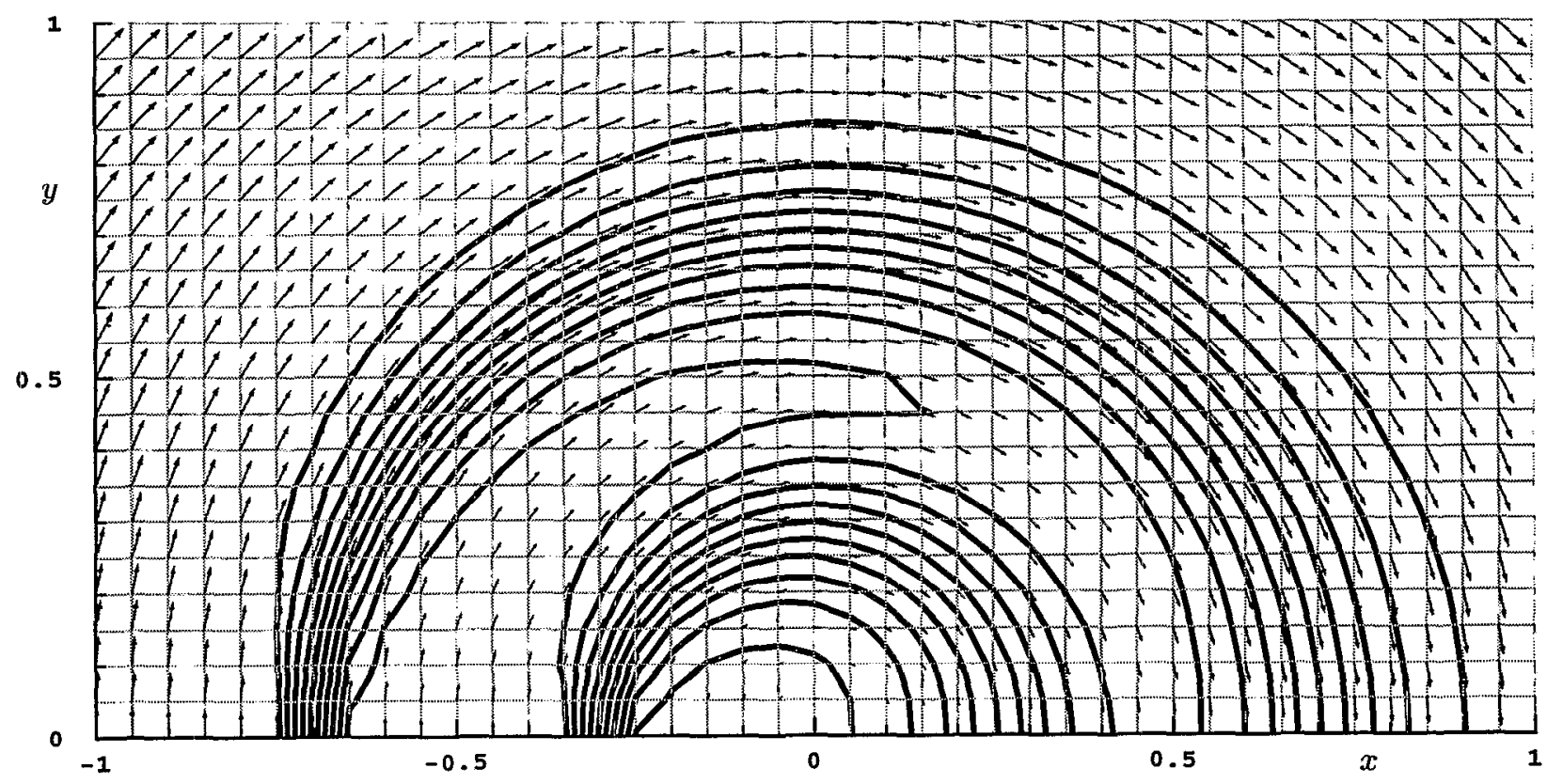

Fig. 7 Circular advection for a Reynolds number of 500 including velocity vectors and solution contours every 0.1 from 0.05 to 0.95 .

\section{Circular Advection}

To remove the added complication of preconditioning a system of equations, the scalar advectiondiffusion model problem was first considered. The case considered here is that of circular advection in the upper half plane, i.e., the domain consisted of 40 equally-spaced cells along the $x$ direction from -1 to 1 and 20 equally-spaced cells along the $y$ direction from 0 to 1 , with a top-hat profile entering at the lower left rotating clockwise to exit at the lower right. Figure 7 shows a representative solution for a Reynolds number of 500 .

The number of iterations to converge the $L_{2}$ error norm to $10^{-10}$ are recorded in Table 1 for a host of Reynolds numbers for both the variablecoefficient Runge-Kutta schome and the constantcoefficient scheme. The fixed-coefficient scheme uses advection-optimized Runge-Kutta coefficients ${ }^{\mathrm{e}}$ due to $\mathrm{T}_{\mathrm{Tai}}{ }^{13}$ for maximizing damping over frequencies from $\pi / 4$ to $\pi$ while the variable-coefficient scheme uses the cell Reynolds number-dependent coefficients that were developed in the preceding section. Note that for this problem the average cell Reynolds numbers are a factor of 20 less than the Reynolds number and actually tend toward zero near the center of the advection velocity field since $R e_{h}=R e h q$. However, for very low values of $q$ we found that the local time-step given by Eq. 7 must be capped to avoid numerical instability regardless of the marching method used.

Table 1 clearly demonstrates the devastating ef-

\footnotetext{
${ }^{\mathrm{e}}$ Coefficients: $\quad \alpha_{1}=0.2131, \alpha_{2}=0.4364, \alpha_{3}=0.7641$ with $\nu=1.1727$.
}

Table 1 Comparison of constant-coefficient RungeKutta scheme to variable-coefficient Runge-Kutta scheme for Circular Advection on a $40 \times 20$ grid.

\begin{tabular}{rrr}
\hline \multirow{2}{*}{$\mathrm{Re}$} & \multicolumn{2}{c}{ Iterations } \\
& Variable & Fixed \\
\hline 1 & 868 & 4,271 \\
10 & 422 & 2,736 \\
25 & 252 & 1,374 \\
50 & 151 & 736 \\
100 & 110 & 387 \\
500 & 85 & 136 \\
1,000 & 77 & 117 \\
5,000 & 81 & 98 \\
10,000 & 82 & 99 \\
\hline \hline
\end{tabular}

fects that low Reynolds numbers (and hence low cell Reynolds numbers) have on the standard advectionoptimized time marching scheme. As anticipated by the Fourier analysis, the variable-coefficient scheme retains a healthy convergence rate even with local cell Reynolds numbers well below 0.01 .

\section{Laminar Boundary Layer}

The flow solver for this portion of the study was purposely kept as simple as possible to isolate the effect of the variable Runge-Kutta coefficients. The solver consists of a cell-centered, finite-volume-based scheme which uses Roe's Flux Difference Splitting ${ }^{29}$ for the inviscid fluxes and central differencing for the viscous 
terms on a structured grid of quadrilaterals. In integral form,

$$
\int_{A} \mathbf{U}_{t} d A+\oint_{S} \mathbf{H} d s=\oint_{S} \mathbf{J} d s
$$

where $\mathbf{U}$ is the conserved state vector defined as $(\rho, \rho u, \rho v, \rho E)^{T}, \mathbf{H}$.is the inviscid flux vector,

$$
(\mathbf{F} \hat{\imath}+\mathbf{G} \hat{\jmath}) \cdot d \vec{s}
$$

and $\mathbf{J}$ is the viscous flux vector,

$$
(\mathbf{R} \hat{\imath}+\mathbf{S} \hat{\jmath}) \cdot d \vec{s}
$$

The Cartesian components of the inviscid flux are

$$
\mathbf{F}=\left(\rho u, \rho u^{2}+p, \rho u v, \rho u H\right)^{T}
$$

and

$$
\mathbf{G}=\left(\rho v, \rho v u, \rho v^{2}+p, \rho v H\right)^{T}
$$

while the viscous components are

$$
\mathbf{R}=\left(0, \tau_{x x}, \tau_{x y}, u \tau_{x x}+v \tau_{x y}-q_{x}\right)^{T}
$$

and

$$
\mathbf{S}=\left(0, \tau_{x y}, \tau_{y y}, u \tau_{x y}+v \tau_{y y}-q_{y}\right)^{T}
$$

The governing equations are non-dimensionalized by freestream speed of sound, $c$, and density, $\rho$, so that the viscous stresses and heat flux terms are given by

$$
\begin{aligned}
& \tau_{x x}=\frac{M}{R e} \mu \frac{2}{3}\left(2 u_{x}-v_{y}\right), \\
& \tau_{y y}=\frac{M}{R e} \mu \frac{2}{3}\left(2 v_{y}-u_{x}\right), \\
& \tau_{x y}=\frac{M}{R e} \mu\left(u_{y}+v_{x}\right), \\
& q_{x}=\frac{M}{(\gamma-1) \operatorname{RePr}} \mu T_{x}, \text { and } \\
& \dot{q}_{y}=\frac{M}{(\gamma-1) \operatorname{RePr}} \mu T_{y}
\end{aligned}
$$

The system is closed with an equation of state,

$$
p=(\gamma-1)\left[F-\frac{1}{2}\left(u^{2}+v^{2}\right)\right]
$$

where $E$ is the total encrgy per. unit volume, $H$ is the total enthalpy per unit volume, $T$ is the temperature, $\gamma$ is the ratio of specific heats (1.4), $\mu$ is the viscosity, $P r$ is the Prandtl number (0.72), and $R e$ is the Reynolds number.

The numerical test problem chosen is that of a laminar boundary layer flow; specifically, two-dimensional subsonic flow over a flat plate. The unit-length Reynolds number is 10,000 and the freestream Mach number is varied between 0.05 and 0.3 . The computational domain and mesh are shown in Fig. 8. The plate is 4 units long, with the upstream boundary 2 units away from the leading edge and the upper boundary is placed at 1.2 units. There are 36 cells evenly distributed along the $x$-direction (24 cells on the plate and 12 upstream) and 40 cells exponentially stretched in the $y$-direction according to

$$
y_{j}=y_{n_{j}} \frac{e^{\varsigma \frac{j-1}{n_{j}-1}}-1}{e^{\varsigma}-1} \quad \text { for } j=1, \ldots n_{j}
$$

where the wall spacing is set to have an acoustic cell Reynolds number of 10 , yielding a stretching parameter, $\varsigma$, of 1.1360..$^{\mathrm{f}}$ By employing characteristic boundary conditions, neither second-order boundary conditions $^{30,31}$ nor solution-assumed grids ${ }^{32-34}$ are necessary to capture the boundary layer gradients (see Fig. 9 where the computed results' ${ }^{5}$ are compared to the Blasius results ${ }^{h}$ ).

Table 2 shows the number of iterations to converge the $L_{2}$ error norm to $10^{-6}$ for a range of Mach numbers for both the fixed-coefficient Runge-Kutta scheme and the variable-coefficient Runge-Kutta scheme. ${ }^{i}$ The

Table 2 Comparison of constant-coefflcient RungeKutta scheme to the variable-coefficient Runge-Kutta scheme for boundary layer flow over a flat plate.

\begin{tabular}{lrrr}
\hline \hline \multirow{2}{*}{$M$} & \multicolumn{2}{c}{ Fixed } & Variable \\
& Uncond. & Precond. & \\
\hline 0.3 & 21,322 & 7,667 & 3,102 \\
0.1 & 45,371 & 7,482 & 1,945 \\
0.05 & 97,263 & 8,231 & 1,543 \\
\hline \hline
\end{tabular}

fixed-coefficient scheme was run with and without preconditioning. The results clearly show the benefits of preconditioning, with local preconditioning providing nearly Mach number independent convergence. However, we note some degradation as the cell Reynolds numbers become lower as the Mach number is lowered. This effect was predicted by D. Lee ${ }^{20,27}$ and shows the limitation of simply using the viscous .Tacobians to augment the Euler preconditioning (see Eq. 18).

Most important, the results for the variablecoefficient scheme show even further improvement in convergence efficiency: the variable-coefficient scheme reaches convergence with nearly a factor of five fewer iterations than the fixed-coefficient scheme.

\footnotetext{
fParabolic stretching, as used in Refs. 30 and 31, was originally tried until, upon further examination, it became apparent that it had a canonical first-point stretching factor of three!

BThe unconditioned and preconditioned solutions are indistinguishable.

${ }^{\mathrm{h}}$ Strictly speaking, Blasius is only valid for incompressible flow, but up to Mach 0.3 compressibility effects are negligible.

${ }^{i}$ CPU timing data are not presented for this proof-of-concept study since no optimization of the new implementation has been attempted.
} 


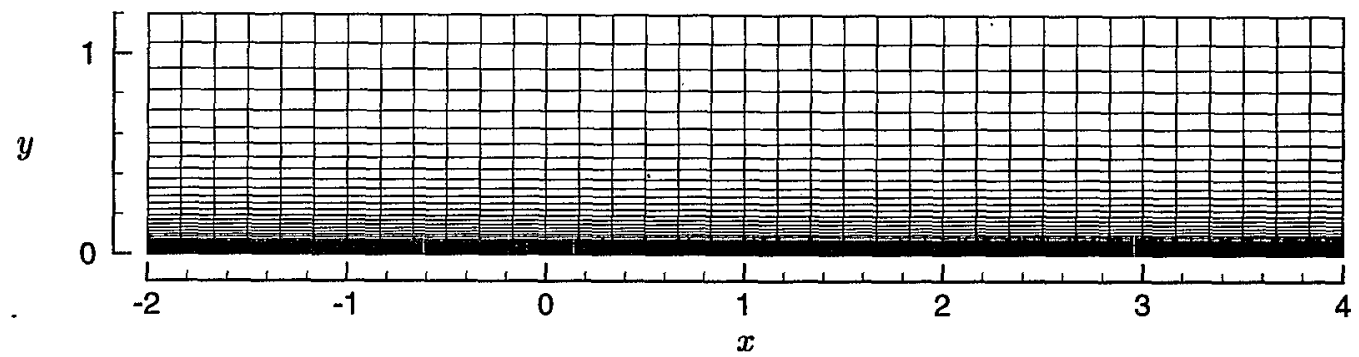

Fig. 8 Computational domain and grid.

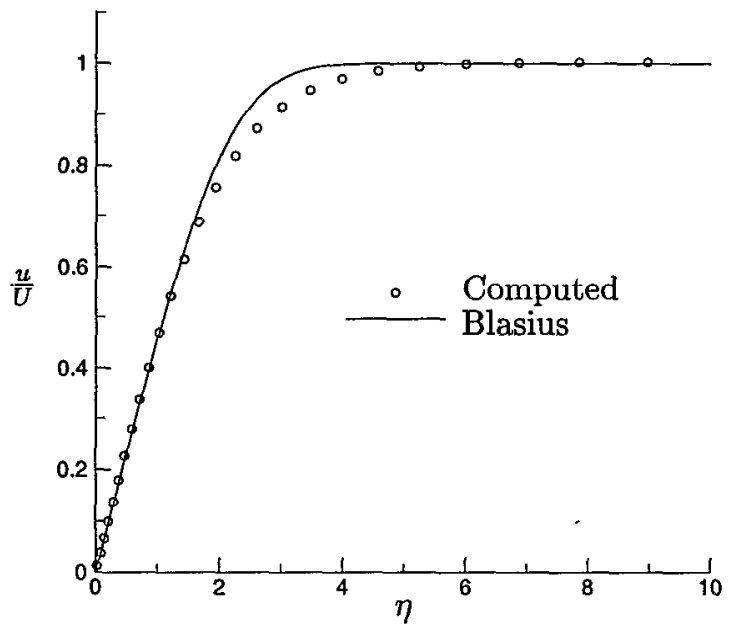

a) $u$-velocity

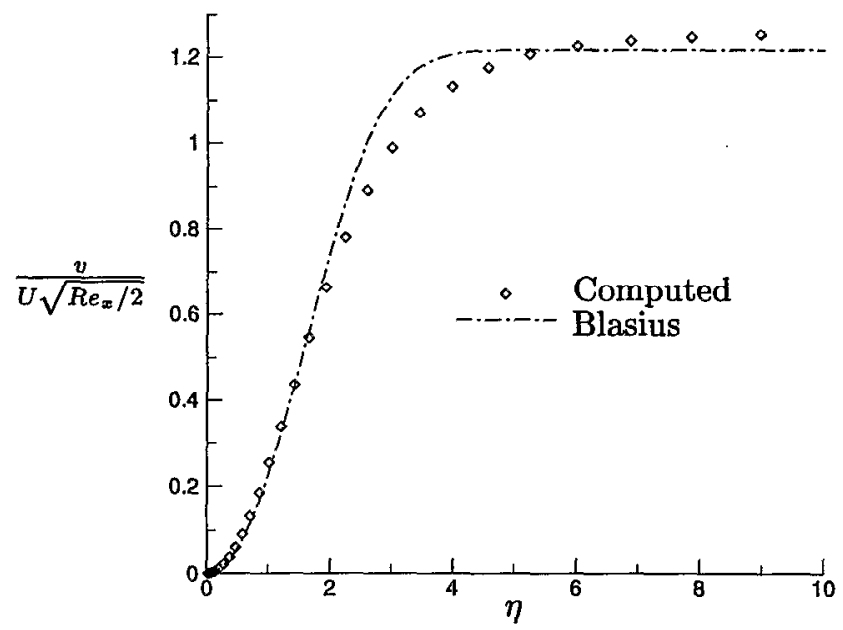

b) v-velocity

Fig. 9 Boundary layer solution profiles at $X / L=0.521$ for Mach 0.1 flow.

\section{Conclusions}

A four-stage time-marching scheme was optimized for a viscous model problem. The Runge-Kutta coefficients as a function of cell Reynolds number were designed to yield a specified damping level while maintaining large time steps. These variable coefficients were applied to a two-dimensional, cell-centered, Navier-Stokes solver which incorporated local preconditioning. The results for a subsonic flat plate boundary layer flow indicate that by employing local RungeKutta coefficients as a function of cell Reynolds number, convergence to steady state is greatly enhanced.

Since this is a proof-of-concept study, many other variables were purposely held constant so that the effects of the cell Reynolds number-dependent RungeKutta coefficients could be ascertained. Given the qualitative improvement over the standard method, the new approach appears to justify further development.

\section{Acknowledgments}

The authors would like to recognize the assistance and discussions with Charlie Swanson, Jeffery White,
Peter Gnoffo, Kyle Anderson, and James Thomas of NASA Langley Research Center, Hampton, Virginia; David Darmofal of MIT University, Cambridge, Massachusetts; Dimitri Mavriplis of ICASE, Hampton, Virginia; Dohyung Lee of NRC, Moffett Field, California; Jens Müller of Oxford University Computing Laboratory, Oxford, United Kingdom; and John Lynn of Ford Motor Co, Dearborn, Michigan.

\section{Colophon}

This paper was typeset in Donald Knuth's 10pt Computer Modern Font using the free, multi-platform ${ }_{\mathrm{A}} \mathrm{T}_{\mathrm{E} X} \mathrm{X}$ typesetting system and Kleb's aiaa bundle. ${ }^{35}$ The psfrag package was used to augment figures generated with TECPLOT ${ }^{\mathrm{TM}}$ while the fix 2 col package was used to correct single and double-column float placement. The nomenclature section was generated automatically with the nomencl package and variable, automatic page referencing was done with the varioref package.

\section{References}

${ }^{1}$ Chorin, A. J., "A Numerical Method for Solving Incompressible Viscous Flow Problems," Journal of Computational 
Physics, Vol. 2, 1967, pp. 12-26.

${ }^{2}$ Choi, D. and Merkle, C. L., "Application of Time-Iterative Schemes to Incompressible Flow," AIAA Journal, Vol. 23, No. 10, Oct. 1985, pp. 1518-1524, (see also AIAA Paper 841638).

${ }^{3}$ Tirkel, F., "Preconditioned Methods for Solving the Incompressible and Low Speed Compressible Equations,". Journal of Computational Physics, Vol. 72, 1987, pp. 277-298.

${ }^{4}$ van Leer, B., Lee, W.-T., and Roe, P. L., "Characteristic Time-Stepping or Local Preconditioning of the Euler Equations," AIAA Paper 91-1552, June 1991.

${ }^{5}$ Courant, R., Friedrichs, K. O., and Lewy, H., "Über die Partiellen Differenzengleichurgen der Mathematischen Physik," Mathematische Annalen, Vol. 100, 1928, pp. 32-74, (see also Ref. 6).

${ }^{6}$ Courant, R., Friedrichs, K. O., and Lewy, H., "On the Partial Difference Equations of Mathematical Physics," IBM Journal, Vol. 5, March 1967, pp. 215-234, (also as AEC Research and Development Report NYO-7689).

${ }^{7}$ Thoman, D. C., Numerical Solutions of Time Dependent Two Dimensional Flow of a Viscous, Incompressible Fluid Over Stationary and Rotating Cylinders, Ph.D. thesis, University of Notre Dame, Aug. 1966.

${ }^{8}$ O'Brien, G. G., Hyman, M. A., and Kaplan, S., "A Study of the Numerical Solution of Partial Differential Equations," Journal of Mathematics and Physics, Vol. 29, No. 4, 1951, pp. 223-249.

${ }^{9}$ Sonneveld, P. and van Leer, B., "A Minimax Problem along the Imaginary Axis," Nieuw Archief voor Wiskunde, Vol. 4, 1985, (see also Delft Report of the Department of Mathematics and Informatics 84-25).

${ }^{10}$ Brandt, A., "Multi-Level Adaptive Solutions to BoundaryValue Problems," Mathematics of Computation, Vol. 31, No. 138, April 1977, pp. 333-390.

11 Jameson, A., "Numerical Solution of the Euler Equations for Compressible Inviscid Fluids," Numerical Methods for the Euler Equations of Fluid Dynamics, edited by. F. Angrand, A. Dervieux, J. A. Désidéri, and R. Glowinski, SIAM, 1985.

${ }^{12}$ van Leer, B., Tai, C.-H., and Powiell, K. G., "Design of Opitmally Smoothing Multi-Stage Schemes for the Euler Equations," AIAA Paper 89-1933, June 1989, (in AIAA 9th Computational Fluid Dynamics Conference proceedings).

${ }^{13}$ Tai, C. H., Acceleration Techniques for Explicit Euler Codes, Ph.D. thesis, University of Michigan, 1990.

${ }^{14}$ Lynn, J. F. and van Leer, B., "Multi-Stage Schemes for the Euler and Navier-Stokes Equations with Optimal Smoothing," AIAA Paper 93-3355, July 1993.

${ }^{15}$ Lynn, J. F. and van Leer, B., "A Semi-Coarsened Multigrid Solver for the Euler and Navier-Stokes Equations with Local Preconditioning," AIAA Paper 95-1667, June 1995.

${ }^{16}$ Lynn, J. F., Multigrid Solution of the Euler Equations with Local Preconditioning, Ph.D. thesis, University of Michigan, 1995.

${ }^{17}$ Choi, Y.-H. and Merkle, C. L., "The Application of Freconditioning in Viscous Flows;" Journal of Computational Physics, Vol. 105, 1993, pp. 207-223, (see also AIAA Paper 91-1652).

${ }^{18}$ Lee, D., Lynn, J. F.; and van Leer, B., "A local NavierStokes Preconditioner for all Mach and Cell Reynolds Numbers," AIAA Paper 97-2024, June 1997.

${ }^{19}$ Lee, D. and van Leer, B., "Progress in Local Preconditioning of the Euler Navier-Stokes Equations," AIAA Paper 93-3328, July 1993.

${ }^{20}$ Lee, D., Local Preconditioning of the Euler and NavierStokes Equations, Ph.D. thesis, University of Michigan, 1996.

${ }^{21}$ Godfrey, A. G., Walters, R. W., and van Leer, B., "Preconditioning for the Navier-Stokes Equations with Finite-Rate Chemistry," AIAA Paper 93-0535, Jan. 1993.

${ }^{22}$ Lorber, A. A., Carey, G. F., Bova, S. W., and Harlé, C., "Accelerated Solution of Non-linear Flow Problems us- ing Chebyshev Iteration Polynomial-based Runge-Kutta Recursions," Computers and Fluids, Vol. 27, No. 7, 1998, pp. 769-782.

${ }^{23}$ Lorber, A. A., Carey, G. F., and Joubert, W. D., "ODE Recursions and Iterative Solvers for Linear Equations," SIAM Journal of Scientific Computing, Vol. 17, No. 1, Jan. 1996, pp. $65-77$.

${ }^{24}$ van Leer, B., "Towards the Ultimate Conservative Scheme. III. Upstream-Centered Finite-Difference Schemes for Ideal Compressible Flow," Journal of Computational Physics, Vol. 23, 1977, pp. 263-275.

${ }^{25}$ van Leer, B., "Upwind-Difference Methods for Aerodynamic Problems Governed by the Euler Equations," Large-Scale Computations in Fluid Mechanics, edited by B. E. Engquist, S. Osher, and R. C. J. Somerville, Vol. 22 of Lectures in Applied Mathematics, American Mathematical Society, 1985, pp. 327-336.

${ }^{26}$ Manteuffel, T. A., "The Tchebychev Iteration for Nonsymmetric Linear Systems," Numerische Mathematik, Vol. 28, 1977, pp. 307-327.

${ }^{27}$ Lee, D., "The Design of Local Navier-Stokes Preconditioning for Compressible Flow," Journal of Computational Physics, Vol. 144, 1998, pp. 460-483, (see also Ref. 20).

${ }^{28}$ Darmofal, D. L. and van Leer, B., "Local Preconditioning: Manipulating Mother Nature to Fool Father Time," Computing the Future II: CFD and Transonic Flow, edited by D. Caughey and M. Hafez, Wiley, (to appear) 1999.

${ }^{29}$ Roe, P. L., "Approximate Riemann Solvers, Parameter Vectors, and Difference Schemes," Journal of Computational Physics, Vol. 43; No. 2, Oct. 1981, pp. 357-372.

${ }^{30}$ Allmaras, S. R., "Analysis of a Local Matrix Preconditioner for the 2-D Navier-Stokes Equations," AIAA Paper 93-3330, July 1993.

${ }^{31}$ Swanson, R. C. and Turkel, E., "Aspects of a HighResolution Scheme for the Navier-Stokes Equations," AIAA Paper 93-3372, July 1993.

${ }^{32}$ Tatsumi, S., Martinelli, L., and Jameson, A., "FluxLimited Schemes for the Compressible Navier-Stokes Equations," AIAA Journal, Vol. 33, No. 2, 1995, pp. 252-261, (see also AIAA Paper 94-0647).

${ }^{33}$ Swanson, R. C., Radespiel, R., and Turkel, E., "On Some Numerical Dissipation Schemes," Journal of Computational Physics, Vol. 147, No. 2, Dec. 1998, pp. 518-544, (see also ICASE Report 97-40 or NASA CR 201726).

${ }^{34}$ Pierce, N. A. and Giles, M. B., "Preconditioning Compressible Flow Calculations on Stretched Meshes," AIAA Paper 95-0889, Jan. 1996.

${ }^{35} \mathrm{Kleb}$, W. L., "aiaa-A IATEX Class and BiBT $\mathrm{T}_{\mathrm{E}} \mathrm{X}$ Style for AIAA Conference Papers and Journal Submission/Simulation," Electronic Documentation, April 1999, Version 2.5.

${ }^{36}$ Richtmyer, R. D. and Morton, K. W., Difference Methods for Initial-Value Problems, Wiley-Interscience, 2nd ed., 1967.

\section{Appendix}

\section{A Non-dimensionalization of the}

\section{Advection-Diffusion Equation}

We begin with

$$
S_{t}+a S_{x}+b S_{y}=\mu\left(S_{x x}+S_{y y}\right)
$$

then define non-dimensional quantities,

$$
\begin{gathered}
\bar{S}=\frac{S}{S_{0}}, \bar{a}=\frac{a}{Q_{0}}, \bar{b}=\frac{b}{Q_{0}}, \bar{\mu}=\frac{\mu}{\mu_{0}}, \\
\bar{x}=\frac{x}{L_{0}}, \bar{y}=\frac{y}{L_{0}}, \text { and } \bar{t}=\frac{t}{L_{0} / Q_{0}} .
\end{gathered}
$$


Substituting into Eq. A.1 yields,

$$
\begin{array}{r}
\frac{\partial\left(S_{0} \bar{S}\right)}{\partial\left(L_{0} / Q_{0} \bar{t}\right)}+Q_{0} \bar{a} \frac{\partial\left(S_{0} \bar{S}\right)}{\partial\left(L_{0} \bar{x}\right)}+Q_{0} \bar{b} \frac{\partial\left(S_{0} \bar{S}\right)}{\partial\left(L_{0} \bar{y}\right)} \\
=\mu_{0} \bar{\mu}\left(\frac{\partial^{2}\left(S_{0} \bar{S}\right)}{\partial L_{0} \bar{x} \partial L_{0} \bar{x}}+\frac{\partial^{2}\left(S_{0} \bar{S}\right)}{\partial L_{0} \bar{y} \partial L_{0} \bar{y}}\right)
\end{array}
$$

Collecting constant terms gives,

$$
\begin{aligned}
\frac{S_{0} Q_{0}}{L_{0}} \bar{S}_{\bar{t}}+\frac{S_{0} Q_{0}}{L_{0}} \bar{a}_{\bar{s}} \bar{S}_{\bar{x}}+\frac{S_{0} Q_{0}}{L_{0}} \bar{b} \bar{S}_{\bar{y}} \\
=\frac{m u_{0} S_{0}}{L_{0}^{2}} \bar{\mu}\left(\bar{S}_{\bar{x} \bar{x}}+\bar{S}_{\bar{y} \bar{y}}\right),
\end{aligned}
$$

while multiplying through by $L_{0} /\left(S_{0} Q_{0}\right)$ and defining $R e=S_{0} L_{0} / \mu_{0}$ yields,

$$
\bar{S}_{\bar{t}}+\bar{a} \bar{S}_{\bar{x}}+\bar{b} \bar{S}_{\bar{y}}=\frac{\bar{\mu}}{R e}\left(\bar{S}_{\bar{x} \bar{x}}+\bar{S}_{\bar{y} \bar{y}}\right) .
$$

If we chose $\mu_{0}=\mu$ and drop the bar notation, we have simply,

$$
S_{t}+a S_{x}+b S_{y}=\frac{1}{R e}\left(S_{x x}+S_{y y}\right) .
$$

B Time-Step Normalization for the

Advection-Diffusion Equation

Let us try to find a non-dimensional time step that includes both advective and diffusive effects.

\section{One-Dimension}

To provide a simple foundation, we begin with the one-dimensional case,

$$
S_{t}+a S_{x}=\frac{1}{R e} S_{x x}
$$

where $S$ is some conserved scalar quantity, $a$ is the advection speed, and $R e$ is Reynolds number. Central differencing for the diffusive term and first-order upwind discretization of the advective term yields,

$$
\mathcal{F}=-\left(\frac{a \Delta t}{\Delta x}+2 \frac{\Delta t}{R e \Delta x^{2}}\right)(1-\cos \beta)-i \frac{a \Delta t}{\Delta x} \sin \beta,
$$

where $\beta$ represents the Fourier frequency. Alternatively, employing a higher-order $\kappa$-scheme ${ }^{24,25}$ for the advective term yields,

$$
\begin{aligned}
\mathcal{F}= & -\left((1-\kappa) \frac{a \Delta t}{\Delta x}+2 \frac{\Delta t}{R e \Delta x^{2}}\right)(1-\cos \beta) \\
& -\frac{(\kappa-1)}{4} \frac{a \Delta t}{\Delta x}(1-\cos 2 \beta) \\
& -i \frac{(3-\kappa)}{2} \frac{a \Delta t}{\Delta x} \sin \beta+i \frac{(1-\kappa)}{4} \frac{a \Delta t}{\Delta x} \sin 2 \beta,
\end{aligned}
$$

where $\kappa \in[-1,1]$.
Typically one defines parameters according to the advective and diffusive terms, e.g.,

$$
\nu=\frac{a \Delta t}{\Delta x} \quad \text { and } \quad \sigma=\frac{\Delta t}{\operatorname{Re} \Delta x^{2}},
$$

where $\nu$ is the Courant number ${ }^{5}$ and $\sigma$ is the Von Neumann number. ${ }^{36}$ This gives a footprint in Fourier space as shown in Fig. B.1 which is an ellipse defined by the Courant number and the Von Neumann number. (The higher-order scheme yields an ellipse only for $\kappa=1$; in general it is egg-shaped.)

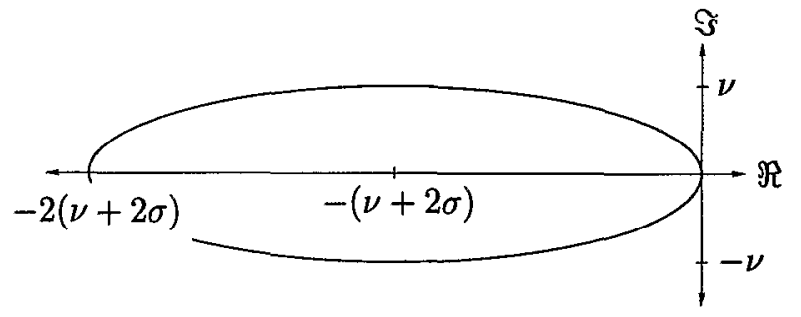

Fig. B.1 Fourier footprint for the one-dimensional Advection-Diffusion equation using first-order upwind spatial discretization for the advective term and central differencing for the diffusion term $(\beta \in[0,2 \pi])$.

Now, suppose we want to fix the location of the negative real extent of this footprint. We can do this by examining the high frequency limit $(\beta=\pi)$ of the Fourier footprint given in Eq. B.2,

$$
-R_{S}=-2\left(\frac{a \Delta t}{\Delta x}+2 \frac{\Delta t}{R e \Delta x^{2}}\right),
$$

where $-R_{S}$ is the specified negative Real axis extent. Now we simply solve for the time step, $\Delta t$,

$$
\Delta t=\frac{\frac{R_{S}}{2}}{\frac{a}{\Delta x}+\frac{2}{R_{e} \Delta x^{2}}} .
$$

For the $\kappa$-scheme the result is similar,

$$
\Delta t=\frac{\frac{R_{S}}{2}}{(1-\kappa) \frac{a}{\Delta x}+\frac{2}{\operatorname{Re} \Delta x^{2}}} .
$$

Thus, by specifying such a time step, the high frequency components of the Fourier footprint remained fixed to $-R_{S}$. We could also write this in terms of a cell Reynolds number, ${ }^{7} R e_{\Delta x}=(a \Delta x) / \mu$ where $\mu=1 / R e$ as shown in Appendix A. For the first-order discretization of the advective term this yields,

$$
\Delta t=\frac{\frac{R_{S}}{2}}{\frac{a}{\Delta x}\left(1+\frac{2}{R e_{\Delta x}}\right)},
$$

or

$$
\Delta t=\frac{R_{S}}{2} \frac{\Delta x}{a} \frac{R e_{\Delta x}}{R \dot{e}_{\Delta x}+2}, .
$$

while the $\kappa$-scheme gives,

$$
\Delta t=\frac{R_{S}}{2} \frac{\Delta x}{a} \frac{R e_{\Delta x}}{R e_{\Delta x}(1-\kappa)+2} .
$$


Alternatively, we could express these as conditions on the Courant and von Neumann numbers,

$$
R_{S}=\left\{\begin{array}{ll}
2(\nu+2 \sigma) & \text { first-order } \\
2[(1-\kappa) \nu+2 \sigma] & \text { higher-order }
\end{array} .\right.
$$

Now, we insert our definition of $\Delta t$ from Eq. B.7 into Eq. B.2 to verify that the footprint is now only dependent on $R_{S}$ and the cell Reynolds number,

$$
\mathcal{F}=-\frac{R_{S}}{2}\left[(1-\cos \beta)+i \frac{R e_{\Delta x}}{R e_{\Delta x}+2} \sin \beta\right] .
$$

So $R_{S}$ controls the negative real extent and the cell Reynolds number dictates the ellipticity.

\section{Two-Dimensions}

While the one-dimensional case is relatively straightforward in terms of parameter choices, etc., adding another dimension creates some "pseudo" ambiguities which are not typically resolved in the CFD community.

The governing equation is now,

$$
S_{t}+a S_{x}+b S_{y}=\frac{1}{R e}\left(S_{x x}+S_{y y}\right) .
$$

Again, using a first-order, upwind approximation for the advective terms and central differencing for the diffusion terms yields,

$$
\begin{aligned}
\mathcal{F}= & -\left(\frac{a \Delta t}{\Delta x}+2 \frac{\Delta t}{R e \Delta x^{2}}\right)\left(1-\cos \beta_{x}\right)-i \frac{a \Delta t}{\Delta x} \sin \beta_{x} \\
- & \left(\frac{b \Delta t}{\Delta y}+2 \frac{\Delta t}{R e \Delta y^{2}}\right)\left(1-\cos \beta_{y}\right)-i \frac{b \Delta t}{\Delta y} \sin \beta_{y}
\end{aligned}
$$

Now there are two Fourier frequency components, $\beta_{x}$ and $\beta_{y}$. The corresponding higher-order result is,

$$
\begin{aligned}
\mathcal{F}= & -\left((1-\kappa) \frac{a \Delta t}{\Delta x}+2 \frac{\Delta t}{R e \Delta x^{2}}\right)\left(1-\cos \beta_{x}\right) \\
& -\frac{(1-\kappa)}{4} \frac{a \Delta t}{\Delta x}\left(1-\cos 2 \beta_{x}\right)-i \frac{a \Delta t}{\Delta x} \sin \beta_{x} \\
& +i \frac{(\kappa-1)}{4} \frac{a \Delta t}{\Delta x} \sin 2 \beta_{x} \\
& -\left((1-\kappa) \frac{b \Delta t}{\Delta y}+2 \frac{\Delta t}{R e \Delta y^{2}}\right)\left(1-\cos \beta_{y}\right) \\
& -\frac{(1-\kappa)}{4} \frac{b \Delta t}{\Delta y}\left(1-\cos 2 \beta_{y}\right) \\
& -i \frac{b \Delta t}{\Delta y} \sin \beta_{y}+i \frac{(\kappa-1)}{4} \frac{b \Delta t}{\Delta y} \sin 2 \beta_{y} .
\end{aligned}
$$

From here there are many avenues to follow, one of the most prevalent is to define component-wise Courant numbers and Von Neumann numbers, e.g.,

$$
\begin{aligned}
& \nu_{x}=\frac{a \Delta t}{\Delta x}, \quad \sigma_{x}=\frac{\Delta t}{R e \Delta x^{2}}, \\
& \nu_{y}=\frac{b \Delta t}{\Delta y}, \quad \text { and } \quad \sigma_{y}=\frac{\Delta t}{R e \Delta y^{2}} .
\end{aligned}
$$

Instead, we propose to express the flow quantities in cylindrical coordinates and the geometric quantities in terms of $\Delta x$ and the cell aspect ratio,

$$
q=\sqrt{a^{2}+b^{2}}, \quad \phi=\arctan \frac{b}{a}
$$

and

$$
\mathbb{R}=\frac{\Delta x}{\Delta y} .
$$

(Cf. Fig. 1 in the main text.) Applying these definitions to Eq. B.13 gives,

$$
\begin{aligned}
\mathcal{F}= & -\left(\frac{q \cos \phi \Delta t}{\Delta x}+2 \frac{\Delta t}{R e \Delta x^{2}}\right)\left(1-\cos \beta_{x}\right) \\
& -i \frac{q \cos \phi \Delta t}{\Delta x} \sin \beta_{x} \\
& -\left(A \frac{q \sin \phi \Delta t}{\Delta x}+2 R^{2} \frac{\Delta t}{R e \Delta x^{2}}\right)\left(1-\cos \beta_{y}\right) \\
& -i R \frac{q \sin \phi \Delta t}{\Delta x} \sin \beta_{y} .
\end{aligned}
$$

As was done for the one-dimensional case, we examine the high frequency limit $\left(\beta_{x}=\beta_{y}=\pi\right)$ of the Fourier footprint given in Eq. B.15,

$$
-R_{S}=-2 \Delta t\left(q \frac{\cos \phi+R \sin \phi}{\Delta x}+\frac{2}{R e} \frac{1+R^{2}}{\Delta x^{2}}\right)
$$

and, solving for the time step such that the negative Real extent of the footprint remains fixed at $-R_{S}$ yields,

$$
\Delta t=\frac{\frac{R_{S}}{2}}{\frac{q \cos \theta}{h}+\frac{2}{R e h^{2}}},
$$

where $h$ is a new length scale that corresponds to the ratio of cell area to the cell's diagonal length and the angle $\theta$ is the angle between $h$ and the flow direction. (Again, cf. Fig. 1.) The $\kappa$-scheme yields a similar form,

$$
\Delta t=\frac{\frac{R_{S}}{2}}{(1-\kappa)^{\frac{q \cos \theta}{h}+\frac{2}{R e h^{2}}} .}
$$

Again, as was done for the one-dimensional case, we can re-write this in terms of a cell Reynolds number, $R e_{h}=(q h) R e$, so Eq. B.17 bccomes,

$$
\Delta t=\frac{R_{S}}{2} \frac{h}{q} \frac{R e_{h}}{R e_{h} \cos \theta+2} ;
$$

and the $\kappa$-scheme yields a similar result,

$$
\Delta t=\frac{R_{S}}{2} \frac{h}{q} \frac{R e_{h}}{R e_{h}(1-\kappa) \cos \theta+2} .
$$

Note the differences with respect to Eq. B.8 and Eq. B.9. In the two-dimensional case we have a new 
length scale, $h$, which amounts to something akin to the harmonic average of the two length scales,

$$
h=\frac{1}{\sqrt{\frac{1}{\Delta x^{2}}+\frac{1}{\Delta y^{2}}}} .
$$

This favors the smaller of the two components. $h$ can also be expressed as the ratio of the cell area to the length of the diagonal,

$$
h=\frac{\Delta x \Delta y}{\sqrt{\Delta x^{2}+\Delta y^{2}}} .
$$

Another twist is that the flow speed component in the " $h$ " direction is what governs the advective portion and not the full flow velocity as one might anticipate.

Now we will substitute our value of $\Delta t$ into the original Fourier footprint of Eq. B.15,

$$
\begin{aligned}
& \mathcal{F}=-\frac{R S}{2} \frac{h}{\Delta x} \frac{R e_{h}}{R e_{h} \cos \theta+2} \\
& {\left[\left(\frac{R e_{\Delta x} \cos \phi+2}{R e_{h}}\right)\left(1-\cos \beta_{x}\right)+i \cos \phi \sin \beta_{x}\right.} \\
&+R\left(\frac{R e_{\Delta x} \sin \phi+2}{R e_{h}}\right)\left(1-\cos \beta_{y}\right) \\
&\left.\quad+i R \sin \phi \sin \beta_{y}\right] . \quad \text { (B.23) }
\end{aligned}
$$

This form is not as straightforward as the onedimensional results, but examining the case of $R=1$, $\phi=45 \mathrm{deg}$, we do recover a similar form;

$$
\begin{aligned}
\mathcal{F}=- & \frac{R_{S}}{2}\left[1-\frac{\left(\cos \beta_{x}+\cos \beta_{y}\right)}{2}\right. \\
& \left.+i \frac{R e_{h}}{R e_{h}+2} \frac{\left(\sin \beta_{x}+\sin \beta_{y}\right)}{2}\right],
\end{aligned}
$$

which for $\beta=\beta_{x}=\beta_{y}$, gives the identical result to Eq. B.11. Figure 2 in the main text shows Fourier footprints for four values of $\kappa$ for the higher-order scheme with $\mathbb{R}=1, \phi=45 \mathrm{deg}$, and $R e_{h}=1$. 